\title{
Mechanical ventilation and tracheostomy in multiple sclerosis
}

\author{
S J Pittock, B G Weinshenker, E F M Wijdicks
}

J Neurol Neurosurg Psychiatry 2004;75:1331-1333. doi: 10.1136/jnnp.2003.024257

Decisions on ventilatory support (VS) in multiple sclerosis (MS) are complex. All patients with MS requiring mechanical ventilation or tracheostomy since 1969 (22) at Mayo Clinic were reviewed. Seventeen had progressive (PMS; 11 secondary and six primary progressive) and one had relapsing remitting MS (RRMS). Four had neuromyelitis optica (NMO). Of those with PMS, all but two required a wheelchair or were bedbound before VS and survived a median of 22 months; 14 were mechanically ventilated and seven underwent subsequent lifelong tracheostomy; three had tracheostomy only. The indications (usually multiple) for VS in PMS patients were aspiration pneumonia, poor ventilation because of mucous plugging, mechanical failure, and airway control/protection for seizures and coma. The RRMS patient required mechanical ventilation for 10 days, with subsequent short-term tracheostomy during a brainstem exacerbation. Of the four patients with NMO one made a dramatic recovery after plasmapheresis. Compared with PMS, the NMO group had a shorter time from disease onset to VS, a longer duration of ventilation, and the three patients not treated with plasma exchange or steroids did worse. The prognosis for independent ventilation ( \pm tracheostomy) was worst for patients with $\mathrm{NMO}$, except for one patient who received plasma exchange, and better then expected for PMS, despite poor preventilation functional status.

$\mathrm{P}$ ulmonary complications are inevitable in many patients with a progressive clinical course of multiple sclerosis (MS) and can be treated. ${ }^{1-4}$ However, when patients become bedbound and reach a terminal stage, the appropriateness and success of ventilatory support is often debated. Recently, we weaned a patient from the ventilator who had a cervical demyelinating lesion after treatment with intravenous methylprednisolone. ${ }^{5}$ There are few studies that look at the usefulness of mechanical ventilation and tracheostomy for MS or other demyelinating diseases of the central nervous system for any indication, including treatment of intercurrent infections. ${ }^{4}$

\section{METHODS}

The medical records of all patients with a diagnosis of MS and neuromyelitis optica (NMO, Devic's syndrome) admitted to the intensive care unit and/or undergoing tracheostomy over the 33 year period ending 2002 were reviewed. Only patients with definite MS (Poser et al criteria) or NMO (Wingerchuk et al criteria) who had mechanical ventilation and/or tracheostomy as a result of their underlying neurological condition were included. ${ }^{67}$

We recorded the course of MS (relapsing remitting (RRMS), secondary progressive (SPMS), and primary progressive (PPMS)), expanded disability status scale (EDSS) before admission, discharge, and last follow up, and reasons for and details of ventilatory support. ${ }^{8}$ We compared groups using the non-parametric Wilcoxon rank sum test and $\chi^{2}$ test where applicable. Criteria for aspiration pneumonia have been proposed by Pugin et al. ${ }^{9}$

\section{RESULTS}

We identified 22 patients who fulfilled our inclusion criteria. Table 1 shows their clinical characteristics.

\section{Progressive MS}

Most patients (17 of 22) had progressive MS (11 had SPMS and six had PPMS). All but two patients with progressive MS were wheelchair bound or worse before admission. Only one patient (EDSS of 6) had a subacute neurological worsening before ventilatory support (previously described). ${ }^{5}$ Of the 17 patients, 14 were treated with mechanical ventilation. Seven underwent subsequent tracheostomy and three had tracheostomy without previous ventilation.

Of the seven patients who underwent tracheostomy after mechanical ventilation, three lived for more than three years, one patient is still alive 26 months later, and three died within four months of weaning off the ventilator. For those patients who were ventilated but did not undergo tracheostomy $(\mathrm{n}=7)$, two lived for more than three years, one lived for 34 months, two are still alive 24 and 116 months after ventilatory support, and two died during mechanical ventilation. There was no significant difference between those who received tracheostomy and those who did not, in respect of age at time of mechanical ventilation (median age, $42 v 50$ years), preventilation functional status (median EDSS, $8.5 v 8.0)$, duration of ventilation (12 $v 7$ days), or survival. Patients who underwent tracheostomy alone tended to be older (median age, 59 years), more disabled (median EDSS, 9) and lived for a shorter time (median, 16 months).

Patients with progressive MS had a shorter median duration of mechanical ventilation ( $8 v 50$ days) compared with patients with NMO. The median survival was two years. However, all patients were severely disabled (median EDSS, 9) and required nursing home care, as they did before the admission to hospital during which they required ventilation.

Ventilatory support was initiated for respiratory failure only $(\mathrm{n}=10)$, airway control/protection only $(\mathrm{n}=3)$, and both of these reasons $(n=4)$. Reasons for airway protection included seizures $(\mathrm{n}=2)$ and coma secondary to sepsis $(\mathrm{n}=5)$. The most common indications for ventilatory support were aspiration pneumonia $(\mathrm{n}=10)$, mucous plugging with difficulty handling secretions $(n=10)$, or both $(\mathrm{n}=13)$. Most patients had multiple reasons for mechanical ventilation.

Abbreviations: CPAP, continuous positive airway pressure; EDSS, expanded disability status scale; MS, multiple sclerosis; NMO, neuromyelitis optica; PPMS, primary progressive multiple sclerosis; RRMS, relapsing remitting multiple sclerosis; SPMS, secondary progressive multiple sclerosis 
Table 1 Clinical characteristics of the 22 patients

\begin{tabular}{|c|c|c|c|c|}
\hline Clinical features & All & RRMS & PMS & NMO \\
\hline Number & 22 & 1 & 17 & 4 \\
\hline $\operatorname{Sex}(F: M)$ & $14: 8$ & $1: 0$ & $10: 7$ & $3: 1$ \\
\hline Age at onset of disease & $35(12-57)$ & 13 & $32(12-57)$ & $40(37-43)$ \\
\hline EDSS before admission or relapse (median (range)) & $8.5(2.0-9.5)$ & 3 & $8.5(6-9.5)$ & $3.5(2.0-8.0)^{*}$ \\
\hline \multicolumn{5}{|l|}{ Type of ventilatory support } \\
\hline Mechanical ventilation only & 9 & 0 & 7 & 2 \\
\hline Ventilation and tracheostomy & 9 & 1 & 7 & 1 \\
\hline Tracheostomy only & 4 & 0 & 3 & 1 \\
\hline $\begin{array}{l}\text { Time from onset to mechanical ventilation/tracheostomy (median years } \\
\text { (range)) }\end{array}$ & $17.5(0.5-32)$ & 9 & $19(8-32)$ & $2.5(0.5-5)^{*}$ \\
\hline Duration of ventilation (median days (range)) & $8.5(1-65)$ & 10 & $8(1-27)$ & $50(5-65)$ \\
\hline Age (years) at time of ventilatory support (median (range)) & $50(22-81)$ & 22 & $51(27-81)$ & $43(38-56)$ \\
\hline \multicolumn{5}{|l|}{ Indication for ventilation/tracheotomy } \\
\hline Respiratory failure only & 13 & & 10 & 3 \\
\hline Airway protection only (seizure or coma) $\dagger$ & 4 & 1 & 3 & \\
\hline Botht & 5 & & 4 & 1 \\
\hline \multicolumn{5}{|l|}{ Underlying cause } \\
\hline Aspiration pneumonia & 12 & 0 & 10 & 2 \\
\hline Mucous plugging & 12 & 1 & 10 & 1 \\
\hline Mechanical respiratory failure (PImax $\leqslant 25 \mathrm{~cm} \mathrm{H}_{2} \mathrm{O}$ ) & 8 & 0 & 4 & 4 \\
\hline \multicolumn{5}{|l|}{ Follow up } \\
\hline Survival: time to death or last follow up (months) & $16(0-91)$ & 17 & $22(0-91)$ & $22(0-60)$ \\
\hline Median time to death after start of ventilation (months) $(n=17)$ & $22(0-91)$ & NA & $22(0-91)$ & $5(0-39)$ \\
\hline Died within 6 months of ventilatory support & 8 & 0 & 6 & 2 \\
\hline Survived $>3$ years after discharge & 10 & 1 & 7 & 2 \\
\hline EDSS at discharge (median (range)) & $9.0(7.5-9)$ & 8.5 & $9(7.5-10)$ & $9(8.5-10)$ \\
\hline Dead at last follow up & 17 & 0 & $14 \ddagger$ & $3 \ddagger$ \\
\hline \multicolumn{5}{|c|}{$\begin{array}{l}\text { * }<0.01 \text {, refers to comparison of NMO with progressive MS group; tunresponsive ( } 5 \text { patients with PMS and } 1 \text { with NMO unresponsive as a result of sepsis, } 1 \\
\text { patient with RRMS and } 1 \text { with NMO unresponsive as a result of brainstem (pontine) inflammation, } 2 \text { patients with PMS and status epilepticus); } ¥ 2 \text { patients with } \\
\text { NMO and } 4 \text { with progressive MS had a necropsy, all were consistent with the clinical diagnosis. } \\
\text { EDSS, expanded disability status scale; MS, multiple sclerosis; NA, not applicable; NMO, neuromyelitis optica; Plmax, maximal inspiratory pressure; PMS, } \\
\text { progressive multiple sclerosis; RRMS, relapsing remitting multiple sclerosis. }\end{array}$} \\
\hline
\end{tabular}

Of the 10 patients who fulfilled criteria for aspiration pneumonia ${ }^{9}$ and eight who required mechanical ventilation, six had subsequent tracheostomy because of inability to wean off the ventilator, despite attempted continuous positive airway pressure (CPAP) or biplane positive airway pressure. Most required CPAP for several days despite tracheostomy. Mucous plugging was identified on bronchoscopy in seven of these 10 patients.

Nine patients had a history of multiple aspiration pneumonias and all who were tested $(\mathrm{n}=8)$ had an abnormal video swallow evaluation and required percutaneous endoscopic gastrostomy tube placement. Four patients had maximal inspiratory pressures $\leqslant 25 \mathrm{~cm} \mathrm{H}_{2} \mathrm{O}$ and complained of dyspnoea at rest, supportive of abnormal respiratory mechanics. Two patients in status epilepticus with a history of MS related seizure disorder required mechanical ventilation and survived for more than five years after discharge.

\section{Neuromyelitis optica}

Four patients had definite NMO (two were necropsy confirmed and the pathology has been described previously $\left.{ }^{10}\right)$. Patients with NMO had a shorter time from onset of symptoms to ventilatory support compared with those with MS (median 2.5 years $v 19$ years; $\mathrm{p}<0.005$ ).

Two patients required ventilatory support in the setting of relapsing myelitis; the first patient, a 38 year old woman, had acute longitudinally extensive inflammation extending from pons to upper cervical cord, required prolonged mechanical ventilation (50 days), and subsequent short-term tracheostomy, and had a dramatic response to steroid and plasma exchange with near full recovery of neurological function. She continued to have relapsing myelitis and optic neuritis and her EDSS score was 5.5 five years after ventilatory support. The second patient, a 42 year old woman, had a prerelapse EDSS of 2.5 and developed pure mechanical respiratory failure and died while being ventilated (65 days). A necropsy confirmed the diagnosis of NMO.

The third, a female patient, with an EDSS of 8, developed respiratory failure secondary to aspiration pneumonia and mucous plugging and required mechanical ventilation for five days; she responded well to antibiotics and survived two years after extubation. No necropsy was performed. The fourth, a man with EDSS 5, survived five months after tracheostomy for progressive mechanical respiratory failure and his necropsy confirmed NMO.

Patients who did not receive plasma exchange or steroids in the NMO group $(n=3)$ fared significantly worse than patients with progressive MS with regard to their median longterm survival (5 months $v 22$ months).

\section{Relapsing remitting $M S$}

A 22 year old woman (treated with interferon $\beta 1$ ) with RRMS developed rapidly progressive diplopia and left arm weakness. Her level of consciousness deteriorated to coma over the course of several days. She was intubated and ventilated for airway protection. She received methylprednisolone $1 \mathrm{~g}$ intravenously for five days and recovered to a "locked in" state. MRI of the head revealed focal confluent signal abnormality in the pons, lower midbrain, and cerebellar peduncles. She received plasma exchange over 10 days, followed by a repeated five day course of methylprednisolone with slow improvement. She was subsequently treated with mitoxantrone and discharged to a rehabilitation facility (EDSS 8.5). She gradually recovered the ability to walk over 17 months.

\section{DISCUSSION}

Little information exists on the indications for and outcome of mechanical ventilation and tracheostomy in MS. Howard et al described 19 patients with MS who had predominantly mechanical respiratory (neuromuscular) failure, 12 of whom required mechanical ventilation. ${ }^{4}$ 
Advanced respiratory support is highly unusual in MS. In our patient registry, we found only 22 patients over a period of 33 years. In our study, most patients with MS who required ventilation or tracheostomy had progressive MS (SPMS > PPMS) and severe disability; only one patient had RRMS. Despite the rarity of NMO in the USA, four patients requiring ventilatory support had NMO. Respiratory failure is a common terminal event in this condition. ${ }^{7}$

The indications for ventilatory support in decreasing order of frequency were aspiration pneumonia, mucous plugging and difficulty handling secretions, mechanical respiratory failure, and coma and status epilepticus. More than one mechanism played a role in most patients. Despite tracheostomy and percutaneous endoscopic gastrostomy tube placement, the risk of further aspiration pneumonia remained high.

Physicians should consider NMO in any patient with a diagnosis of MS who presents with mechanical respiratory failure. Half of the eight patients in our study with documented unsatisfactory inspiratory pressures had NMO.

Recent studies suggest that the spectrum of NMO may extend beyond the traditional requirement for both optic nerve and spinal cord involvement, to include single or longitudinally extensive myelitis that may also involve the brainstem (as in one of our cases), and in some cases with coexistent abnormalities in visually evoked potentials.

Survival in the patients with progressive MS was better then expected, despite their poor premechanical ventilation functional status. Other patients admitted to the intensive care unit with MS related respiratory problems may have chosen not to be ventilated and probably died.

The decision to provide respiratory support to severely debilitated patients with MS is a difficult one. Our study suggests that although there may be a worsening in disability, patients with MS who receive ventilatory support may have longer survival (median of two years). This conclusion should be considered in the context of its impact on quality of life as a result of worsening disability.

\section{Authors' affiliations}

S J Pittock, B G Weinshenker, E F M Wijdicks, Department of Neurology Mayo Clinic, Rochester, MN 55905, USA

Competing interest: none declared

Correspondence to: Dr E F M Wiidicks, Department of Neurology, Mayo Clinic, W8B, 200 First Street SW, Rochester, MN 55905, USA; wijde@mayo.edu

Received 23 July 2003

In revised form 18 November 2003

Accepted 25 November 2003

\section{REFERENCES}

1 Carter JL, Noseworthy JH. Ventilatory dysfunction in multiple sclerosis. Clin Chest Med 1994;15:693-703.

2 Buyse B, Demedts M, Meekers J, et al. Respiratory dysfunction in multiple sclerosis: a prospective analysis of 60 patients. Eur Respir J 1997;10:139-45.

3 Gosselink R, Kovacs L, Decramer M. Respiratory muscle involvement in multiple sclerosis. Eur Respir J 1999;13:449-54.

4 Howard RS, Wiles CM, Hirsch NP, et al. Respiratory involvement in multiple sclerosis. Brain 1992;115:479-94.

5 Pittock SJ, Rodriguez M, Wijdicks EFM. Rapid weaning from mechanical ventilator in acute cervical cord multiple sclerosis lesion after steroids. Anaesth Analg 2001;93:1550-1.

6 Poser CM, Paty DW, Scheinberg L, et al. New diagnostic criteria for multiple sclerosis: guidelines for research protocols. Ann Neurol 1983;13:227-31.

7 Wingerchuk DM, Hogancamp WF, O Brien PC, et al. The clinical course of neuromyelitis optica (Devic's syndrome). Neurology 1999;53:1107-14.

8 Kurtzke JF. Rating neurologic impairment in multiple sclerosis: an expanded disability status scale (EDSS). Neurology 1983;33:1444-52.

9 Pugin J, Auckenthaler R, Mili N, et al. Diagnosis of ventilator-associated pneumonia by bacteriologic analysis of bronchoscopic and nonbronchoscopic "blind" bronchoalveolar lavage fluid. Am Rev Respir Dis 1991;43:1121-9.

10 Lucchinetti CF, Mandler RN, Mcgavern D, et al. A role for humoral mechanisms in the pathogenesis of Devic's neuromyelitis optica. Brain 2002; 125:1450-61. 